First publ. in: Flavins and flavoproteins 1990 : proceedings of the tenth International Symposium, Como, Italy, July 15 - 20, 1990, pp. 325-328

\title{
ON THE ROLE OF GLU 376 IN CATALYSIS OF ACYL-COA DEHYDROGENASES
}

Kathrin Ankele, Klaus Melde, Stefan Engst, Peter Bross, Sandro Ghisla

University of Konstanz PO.BOx 55607750 Konstanz FRG

Arnold W. Strauss

Washington University School of Medicine, St. Louis, Missouri 63110, USA

Introduction:

Acyl-COA dehydrogenases are flavoproteins involved in the degradation of fatty acids and of branched chain amino acids. Their reaction mechanism is assumed to involve a concerted

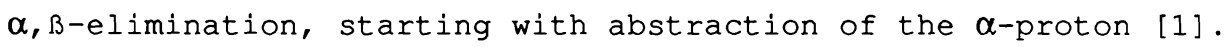
Incubation of medium and short chain acyl-COA dehydrogenases (MCADH and SCADH) with 2-octynoyl-COA leads to covalent modification of the enzyme active site $[2,3]$. The amino acid involved is $\mathrm{Glu}^{376}$, which has been proposed to be the base abstracting the hydrogen as an $\alpha$-proton [4]. Since Glu 376 is not conserved in all acyl-COA dehydrogenases (Isovaleryl-COA and LCADH have Gly at position 376) [5], the above mentioned role of $\mathrm{Glu}^{376}$ can be questioned. In order to investigate the role of Glu376, we studied the reactivity with 2-octynoyl-COA of two enzymes lacking Glu376. These are long chain acyl-COA dehydrogenase (LCADH) which has a Gly 376 [5], and the Glu ${ }^{376}-\mathrm{Gln}$ mutant of human $\mathrm{MCADH}$ [6].

Results and Discussion:

Reaction of 2-octynoyl-COA with LCADH: The incubation of LCADH with 2-octynoyl-CoA leads to changes of the oxidized flavin spectrum which are similar to those reported by Freund et al.[3] using MCADH. A difference is the complete 
disappearance of the charge-transfer complex absorption in our case (see Figure 1). Importantly, however, in the present case the enzyme activity could be partially restored ( $50 \%)$ after ultrafiltration. This indicates that 2-octynoyl-COA does not lead to covalent modification of the active site, although it is being acted on by the enzyme. The product acts as a strong competitive inhibitor, thus anaerobic incubation of 6 equivalents of octanoyl-COA with enzyme which had been inactivated with 2-octynoyl-COA and then ultrafiltered leads to complete reduction of the flavin spectrum within a few minutes (data not shown).

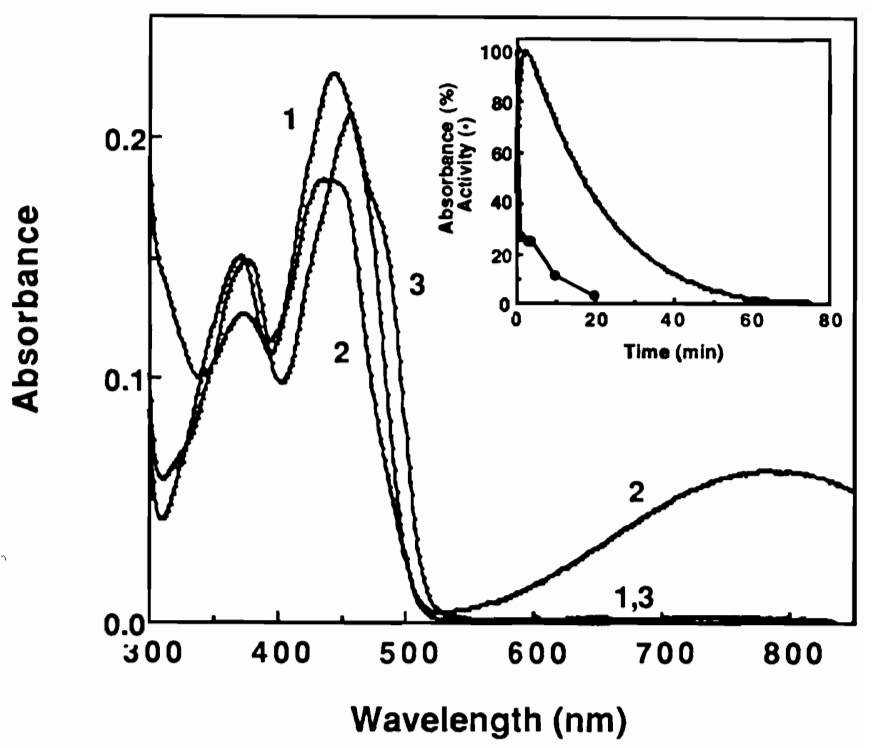

Figure 1:Spectral changes observed upon incubation of LCADH with 2.4 equivalents of 2-octynoyl-COA in $100 \mathrm{~mm}$ phosphate, $\mathrm{pH} 8,25^{\circ} \mathrm{C}$. Curves 1-3: spectra recorded at $0 \mathrm{~min}$. (1), $3 \mathrm{~min}$. (2), and $75 \mathrm{~min}$. (3). The inset shows the formation and decay of the charge transfer complex absorption at $800 \mathrm{~nm}$ and the activity of the enzyme at the times shown. Note that $\sim 50 \%$ activity could be regained after ultrafiltration. 
Reaction of 2-octynoyl-CoA with human Glu ${ }^{376}-$ Gln MCADH mutant: Incubation of this human MCADH mutant with 3 equivalents of 2-octynoyl-CoA leads to spectral changes which are significantly different to those observed upon incubation with MCADH or LCADH.

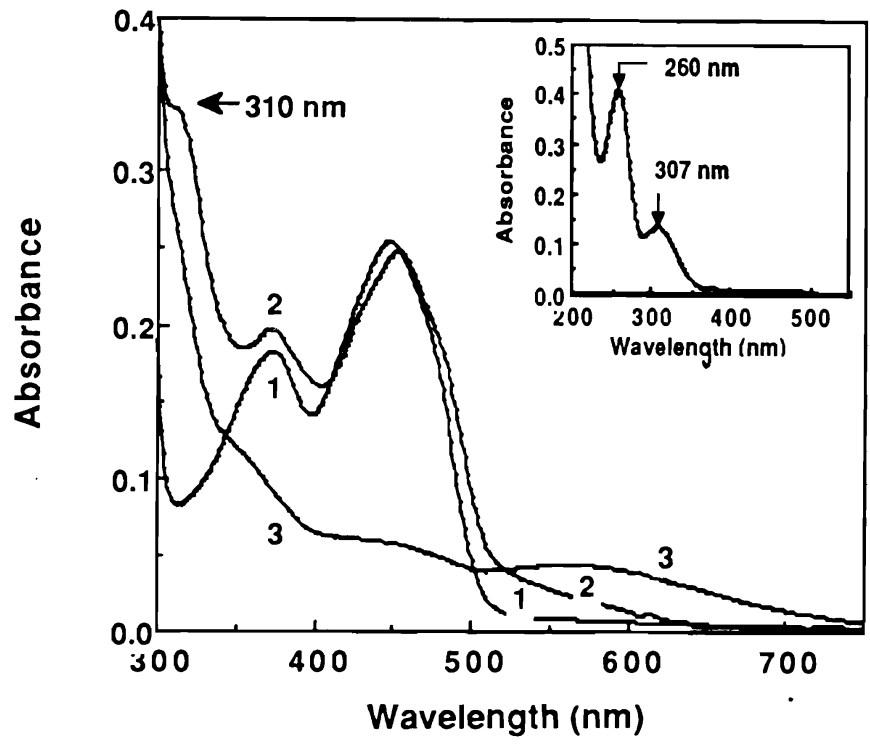

Figure 2: Spectral properties of the Glu ${ }^{376}-$ Gln mutant of human MCADH before (1) and after $17 \mathrm{~h}$ of aerobic incubation with 3 equivalents of 2-octynoyl-CoA (2). Spectrum (3) represents the reduced enzyme (2) after $20 \mathrm{~h}$ of anaerobic incubation with 6.7 equivalents of octanoyl-COA. The inset shows the spectral properties of the product of human Glu ${ }^{376}-\mathrm{Gln}$ MCADH mutant incubation with 2-octynoyl-CoA after ultrafiltration in $100 \mathrm{~mm}$ phosphate, $25 \mathrm{mM} \mathrm{MgCl} 2, \mathrm{pH}$. At $\mathrm{pH} 5$ and 9 the spectrum is unchanged (not shown).

Immediately after addition, the red-shift of the $450 \mathrm{~nm}$ band typical for binding of COA-derivatives to the enzyme occurs (not shown). After $20 \mathrm{~h}$ a distinct shoulder at $310 \mathrm{~nm}$ and an absorption at around $550 \mathrm{~nm}$ has developped as shown in figure 2. This absorption is probably due to formation of a charge 
transfer complex and is similar to what is observed upon incubation of human MCADH mutant with 3-oxoacyl-CoA derivatives (data not shown). The absorbance at $310 \mathrm{~nm}$ was decreased by ultrafiltration. The spectrum of the product, present in the filtrate, is compatible with a CoA-thioester ( $\lambda_{\max } 260 \mathrm{~nm}$ ). However, since changes in the $\mathrm{pH}(\mathrm{pH} 5,7$ and 9) have only little effect on the spectrum, it is unlikely that it is a 3-oxoacyl-COA ester. The enzyme recovers the ability to be reduced by octanoyl-COA.

Conclusions:

Our results, together with those described elsewhere in this volume for the Glu376-Gln mutant [7], strongly suggest that this Glu is an important, but not necessary residue for catalysis of $\alpha, \beta$-dehydrogenation in some acyl-CoA dehydrogenases. If its role is taken over by another residue in $\mathrm{LCADH}$, then this residue obviously is incapable of reacting with the products arising from octynoyl-COA to form a covalent adduct.

References:

1.Ghisla, S. 1984. In: Flavins and Flavoproteins (R.C. Bray, P.C. Engel and S.G. Mayhew eds.) Walter de Gruyter, Berlin New York, p.385.

2.Fendrich, G., R. H. Abeles. 1982. Biochemistry 21, 6685

3.Freund, K., J.Mizzer, W. Dick, C.Thorpe. 1985. Biochemistry $\underline{24}, 5996$.

4.Powell, P.J., C. Thorpe. 1988. Biochemistry 27, 8022.

5. Matsubara, Y., Y. Indo, E. Naito, H. Ozasa, R. Glassberg, J. Vockley, Y. Ikeda, J. Krauss, K. Tanaka, K.. 1989. J. Biol. Chem. 264, 16321 .

6.Bross, P., S. Engst, A.W. Strauss, D.P. Kelly, I. Rasched, S. Ghisla. 1990. J.Biol.Chem. 265, 7116.

7.Engst, S.,P. Bross, J. Stiemke, A. Schieber, A.W. Strauss, D. Kelly, I. Rasched, S. Ghisla (same issue). 